\title{
1 SURVIVAL OF HORSES FOLLOWING STRANGULATING LARGE COLON
}

2 VOLVULUS

3

4 Keywords: horse, large colon volvulus (LCV), strangulating, survival, colic.

5 Word Count: 4638 


\section{Summary}

7 Reasons for performing study: The pattern of long-term survival and specific factors associated with long-term survival have not previously been evaluated in horses with a

9 strangulating large colon volvulus (LCV).

10 Objectives: The aims of this study were to provide data on the long-term survival of horses with LCV and to identify pre-, intra- and post-operative variables associated with survival.

Methods: Clinical data and long-term follow up information were obtained from 116 horses with a strangulating LCV $\left(\geq 360^{\circ}\right)$ undergoing general anaesthesia. Two multivariable Cox proportional hazards models for post-operative survival time were developed: Model one included all horses and evaluated pre-operative variables and model two included horses that survived anaesthesia and evaluated pre, intra and post-operative variables.

Results: The study population comprised 116 horses. Eighty-nine (76.6\%) survived general anaesthesia. Of these, the percentage that survived until discharge, to one year, and to two years was $70.7 \%, 48.3 \%$ and $33.7 \%$ respectively. Median survival time for horses that survived general anaesthesia was 365 days. In model one increased pre-operative packed cell volume (PCV) was significantly associated with reduced post-operative survival (Hazard Ratio [HR] 1.08, 95\% confidence intervals [CI] 1.05 - 1.11). However, this effect changed over time. In model two abnormal serosal colour intra-operatively (HR 3.61, 95\% CI 1.55 8.44), increased heart rate at 48 hours post-surgery (HR 1.04, 95\% CI $1.02-1.06$ ), and colic during post-operative hospitalisation (HR 2.63, 95\% CI $1.00-6.95$ ), were all significantly associated with reduced post-operative survival.

27 Conclusions: Survival time in horses with a LCV was associated with pre-operative PCV, serosal colour, heart rate at 48 hours post-operatively, and colic during post-operative hospitalisation. 
Potential relevance: This study provides evidence based information on the long-term survival of horses with LCV and identifies parameters that may assist decision making by clinicians and owners.

\section{Introduction:}

Identification of factors that are significantly associated with prognosis following surgical treatment of colic allows clinicians and owners to make informed decisions regarding the management of individual horses $[1,2]$. It has been recognised that these key prognostic indicators, and survival patterns following surgery, differ for various types of colic $[1,2,3]$. However, relatively few studies provide information regarding survival patterns following hospital discharge or on key prognostic indicators for specific surgical lesions.

Large colon volvulus (LCV) is one of the most painful and rapidly fatal causes of colic in the horse [4]. LCV represents between 10 and $20 \%$ of horses with colic that undergo exploratory laparotomy [5,6]. Volvulus greater than or equal to 360 degrees has been found to be strongly associated with poor survival [3], with survival to discharge following strangulating LCV reported to lie between 36 and $74 \%$ [7, 8, 9]. In a study modelling long-term survival of horses following surgery for large intestinal disease, age, pre-operative PCV and heart rate were found to be significantly associated with post-operative mortality; however, horses with a strangulating LCV represented only $20 \%$ of the cases included in this study [3]. Postoperative persistent tachycardia has been previously suggested to indicate a poor prognosis

51 for survival in horses with LCV [10] and in horses undergoing large colon resection and 52 anastomosis (LCRA), increased heart rate at 24 hours post-operatively was significantly associated with post-operative mortality [11]. However, specific determinants of survival for 
54 horses with a strangulating LCV have not been investigated in a large number of cases using survival analysis.

57 The aims of this study were to provide data on the long-term survival of horses with strangulating LCV and to identify pre, intra and post-operative variables associated with survival.

60

\section{Materials and methods:}

\section{Study population}

63 The case records of all horses with a LCV, (greater than or equal to 360 degrees), identified 64 at exploratory laparotomy between $1^{\text {st }}$ January 2001 and $31^{\text {st }}$ December 2010 at the detail to 65 be provided on acceptance Equine Hospital, United Kingdom were reviewed. Horses were 66 included if they underwent general anaesthesia and exploratory laparotomy. Horses that were euthanased during surgery were included, but horses that died or were euthanased prior to anaesthesia were excluded from the study population. Pre-, intra- and post-operative data were extracted from hospital records and entered into a computer database. Short-term survival data whilst in the hospital (reason for death and date of death) were obtained from hospital records. To evaluate long term survival, telephone questionnaires with owners were conducted following discharge from the hospital. The telephone questionnaire asked the date of death and reason for death. Questionnaires were conducted quarterly for the first year following colic surgery and bi-annually thereafter as part of an on-going study on colic survival [3]. This was with the exception of a 30-month period during which questionnaires were suspended due to an alteration in the study's funding. Owners were re-contacted after this period. All horses remained in the study until they died or were lost to follow up, for 
example, following a change of ownership. In cases where the owner was unable to provide the exact date of death or censorship, this was defined as the mid-point of the week or month in which the horse was reported to have died or been sold. In this study the term death includes death due to euthanasia.

Statistical analysis

84 Descriptive data were generated and survival time was used to construct a Kaplan-Meier plot of cumulative probability of survival [12]. The study population was divided into two groups.

Group one included the entire study population and evaluated pre-operative variables (model one). Group two included only those horses that recovered successfully from anaesthesia and evaluated pre, intra and post-operative variables (model two). Prior to univariable analysis, all variables were assessed for correlation using Spearman's rank correlation coefficients.

90

For both groups association with survival time was modelled using Cox proportional hazards models [12]. Survival time (days) was measured as a continuous variable starting from the date of induction of anaesthesia until death or censoring. Potential explanatory variables were screened for univariable association with survival time. The functional form of the relationship between continuous variables and survival time was modelled using penalised Cox models and by generating smoothing splines for each continuous variable [13]. Variables showing some evidence of univariable association with outcome $(\mathrm{P}<0.2)$ were evaluated in a multivariable Cox proportional hazards model, which was constructed using a backwards,

99 stepwise elimination procedure [12]. Variables remained in the model if they significantly 100 improved the fit $(\mathrm{p} \leq 0.05)$ assessed using the likelihood ratio statistic. Variables with $>33 \%$ 101 of missing values were excluded from the initial model-building procedure. Biologically 
102 plausible interaction terms for variables remaining in the final model were assessed. As Cox

103 models assume proportional hazards, (i.e. the effect of a variable on the outcome is constant

104 over time), model diagnostics performed included Schoenfield residual plots to assess

105 proportionality throughout survival time. In addition, graphical assessment of log cumulative

106 hazards plots for categorical variables was performed. Scaled changes in the regression

107 coefficient for each observation (delta betas) were used to evaluate potential leverage by

108 individual observations for each variable [12]. The model was rerun excluding observations

109 with large delta-beta values $(>0.4$ or $<-0.4)$ to evaluate their influence on parameter

110 estimates. The critical probability for all analyses was set at 0.05 .

112 Results:

113 The study population included 116 horses, of which $63(54 \%)$ were mares, 52 (45\%) were 114 geldings and 1(1\%) was a stallion. The median age of horses was 11 years (range 1-28 years) 115 and the median weight was $580 \mathrm{~kg}$ (range $350-750 \mathrm{~kg}$ ). The median duration of colic signs

116 prior to surgery was 10 hours (range 3-96 hours). Median heart rate on admission was 60 117 beats/min (range 32-108 beats/min) and median packed cell volume (PCV) was 45\% (range 118 27-65\%). Only two horses underwent a large intestinal resection.

120 Of the 116 horses, $89(76.7 \%)$ recovered from anaesthesia. Of these horses, the percentage 121 that survived until discharge, to one year, and to two years was $70.8 \%, 48.3 \%$ and $33.7 \%$ 122 respectively. The reasons for death are shown in table 1. Sixteen horses were lost to follow 123 up. The study included 67,386 days of survival. Median survival time for all horses was 88 124 days (Figure 1a) and median survival time for those horses that successfully recovered from anaesthesia was 365 days (Figure 1b). 
127 The results of univariable relationships of potential explanatory pre-operative variables with 128 survival time as the outcome measure, when all the horses were included (Group one) are

129 shown in Supporting items 1 and 2 (available online). On univariable analysis pre-operative

130 variables significantly associated with increased risk of post-operative death were the degree

131 of pain on admission, pre-operative heart rate and pre-operative PCV. The final Cox

132 proportional hazards model (Model one) included only the variable pre-operative PCV,

133 which was positively and significantly associated with the risk of post-operative death and

134 demonstrated a linearly increasing likelihood of mortality (Figure 2 and Table 2). However,

135 there was evidence of significant non-proportionality of the effect of the variable PCV over

136 time (Figure 3), with reduced risk over time $(\mathrm{p}<0.007)$. A PCV*In(time) interaction term was

137 added to the model (Table 2). The effect of PCV was allowed to interact with time on a

138 natural $\log$ scale; this was chosen since it was assumed that the effect of PCV would be

139 greatest in the immediate post-operative period and then reduce as time passed. The

$140 \mathrm{PCV} * \ln ($ time$)$ interaction term was significant, confirming that the effect of PCV does vary

141 with time. Using this model, the hazard ratio was computed at a number of time points, with

142 the hazard ratio at time $t$ equal to $1.08^{*} 0.96^{\ln (t)}$ (Table 3 ). The effect of pre-operative PCV

143 decreases until day 7 post-operatively then the effect disappears.

145 To illustrate these results, a horse with a LCV that has a PCV on admission of 55\% has a 4.7

146 times greater risk of mortality, intra or post-operatively, than a horse with a pre-operative

147 PCV of $35 \%$. 
149 The results of univariable relationships of potential explanatory pre, intra and post-operative

150 variables with survival time as the outcome measure, when only those horses that recovered 151 successfully from anaesthesia were included (group two) are shown in Supporting items 3 152 and 4 (available online). Categorical variables that were significantly associated with 153 increased risk of post-operative death were colour of large colon serosa (normal/abnormal),

154 colour of mucosa at the enterotomy site (normal/abnormal), haemorrhage at the enterotomy 155 site (present/absent), colic during post-operative hospitalisation (no/yes) and repeat 156 laparotomy (no/yes). Derangements in serosal and mucosal colour and the presence of 157 haemorrhage at the enterotomy site were defined by the surgeon at the time of exploratory 158 laparotomy and recorded in the horse's surgical report. Continuous variables significantly 159 associated with increased risk of post-operative death were pre-operative heart rate and pre160 operative PCV, heart rate at 24 and 48 hours post-operatively, PCV at 24 and 48 hours post161 operatively and total protein at 24 and 48 hours post-operatively. The final Cox proportional hazards model included serosal colour intra-operatively (normal/abnormal), heart rate at 48

163 hours post-surgery and colic during post-operative hospitalisation (no/yes), which were all 164 positively associated with risk of post-operative death (Table 4). Examination of the 165 functional form of the relationship of heart rate at 48 hours post-operatively demonstrated a 166 linearly increasing likelihood of mortality, with no evidence of non-linearity (Figure 4). In 167 model two graphical and statistical evaluation of Schoenfeld residuals for all variables, and 168 graphical assessment of $\log$ cumulative hazard plots for categorical, confirmed the 169 assumption of proportional hazards to be valid (Supporting item 5 and Supporting item 6a 170 and Supporting item 6b, available online). 
172 To illustrate the results for model two, a horse with a LCV that has a heart rate of 60 beats

173 per minute at 48 hours post-operatively has a 2.2 times greater risk of post-operative

174 mortality than a horse with a heart rate of 40 beats per minute at 48 hours post-operatively.

175

176 For both models removal of influential individual observations with large delta-betas had

177 little effect on coefficients, showing the models were stable and all observations were

178 retained within the models.

179

180 Discussion

181 This study provides information on survival following surgery for a specific type of colic 182 (strangulating LCV) and identifies risk factors for post-operative survival. The results of the 183 study may assist clinicians with decision-making in the management of horses with 184 strangulating LCV and clinicians can utilise this information to provide owners of these 185 horses with evidence-based information regarding their survival following surgery.

187 Survival to discharge following LCV has been previously reported to lie between 36-74\% [7,

$1888,9]$. This wide range can partially be attributed to differences between hospital populations, 189 the lack of consistency in the definition of a strangulating LCV and the fact that some studies 190 excluded horses that did not survive anaesthesia. Variation in the number of horses 191 euthanised without an opportunity for exploratory laparotomy, due to a poor prognosis, will 192 also influence survival rate. This study included only horses with a volvulus of greater than 193 or equal to 360 degrees in order to ensure all cases of volvulus were strangulating in nature.

194 LCV was found to be associated with considerable post-operative mortality, with over $20 \%$ 195 of horses not recovering from anaesthesia. Of those horses that recovered from anaesthesia, 
196 less than 50\% were alive one year post-operatively and only a third of horses were alive two

197 years post-operatively.

198

199 Two models were created to allow exploration of pre, intra and post-operative parameters. In

200 model one only increased pre-operative packed cell volume (PCV) on admission was

201 significantly associated with increased post-operative mortality. This significant association

202 emphasises the importance of early referral of horses with a suspected LCV, prior to

203 alteration of their PCV. Pre-operative PCV has been shown previously to be significantly

204 associated with long term survival for other colic types [1,2] and in particular with long term

205 survival following surgery for large intestinal disease [3]. Pre-operative PCV was found to be

206 associated with an increased risk of mortality in the early post-operative period and its

207 influence diminished by day 7. Elevated PCV on admission is likely to reflect the degree of

208 systemic inflammatory response that may develop during hospitalisation, which will affect

209 survival in the immediate post-operative period. Pre-operative PCV was not retained in

210 model two when intra and post-operative variables were included. It is likely that those

211 horses that died or were euthanased on the operating table (and were therefore excluded from

212 model two), had a high PCV on admission, and were excluded from model two.

213

214 When pre, intra and post-operative variables were included in a multivariable model, the

215 variables abnormal serosal colour of the large colon intra-operatively, heart rate at 48 hours

216 post-operatively, and colic during post-operative hospitalisation were found to be

217 significantly associated with reduced long term survival. Alteration in serosal colour is

218 indicative of the degree of vascular compromise of the large colon. It may correlate with the

219 degree of the systemic inflammatory response, which occurs as a result of the loss of mucosal 
220 barrier function, and the subsequent trans-mural and trans-vascular migration of microbes

221 and pathogen associated molecular patterns, including LPS (endotoxin), into the peritoneal cavity and circulation $[14,15]$. Alternative intra-operative methods of estimating prognosis in cases of LCV, including pulse or surface oximetry, fluorometric evaluation $[16,17]$ and colonic luminal pressure [18] are often unreliable and may not be readily available. Histopathologic evaluation of an intra-operative biopsy, collected from the pelvic flexure, examining the crypts and interstitial crypt space has been shown to be a highly sensitive and specific predictor of post-operative survival in cases of LCV $[19,20]$. However, intraoperative evaluation of a biopsy is often impractical and a more recent study found histopathological evaluation of pelvic flexure biopsies did not accurately predict survival [21].

232 Post-operative persistent tachycardia has been suggested to indicate a poor prognosis for survival in horses with LCV [10]; however, its effect has never previously been quantified. Post-operative tachycardia is related to the degree of systemic inflammatory response present post-operatively [22], which is likely to determine survival in cases of strangulating LCV. Colic during post-operative hospitalisation may be due to localised sites of ischaemia,

237 adhesion formation or stenosis [23]. Physiological derangements of the large intestine may predispose certain individuals to recurrent LCV or large colon displacement [24]. The risk of post-operative colic is significantly increased in horses with LCV [23] and in this study

240 nearly $20 \%$ of this population died as a result of colic following discharge from the hospital.

241 Colic during hospitalisation may contribute to a decision for euthanasia during hospitalisation

242 or in other cases be a marker for colic post discharge from the hospital. Further investigation into the incidence of post-operative colic in horses with LCV is warranted. 
245 Variables with over $33 \%$ of missing values were excluded from the initial model building 246 procedure. Whilst much of the data included in this study was collected prospectively some 247 data was missing due to the severity of pain on admission and the obvious requirement for 248 immediate exploratory laparotomy. Adequate and complete follow-up is a prerequisite for the 249 conduct of any survival study [25]. The 30-month period during the study where follow-up 250 questionnaires were suspended was due to an alteration in funding, and may have reduced the accuracy of survival time and increased the number of censored individuals. In addition, as in numerous other veterinary studies evaluating survival time, the censorship of horses that were lost to follow-up, and the lack of differentiation between death and euthanasia, may have resulted in erroneous inferences from the data [26, 27]. Horses that were euthanased on the operating table were not excluded from the study population; it was deemed their inclusion would provide clinicians and owners with more accurate information on the prognosis of horses presenting with a strangulating LCV. However, the inclusion of horses that were euthanased on the operating table allows a surgeon's judgment and the owner's financial situation to have a potential influence on survival time. In an attempt to assess the effect of financial constraints on survival, insurance status was assessed within the models, and was found not to be significant (see Supporting items 1 and 3).

263 Extrapolation of these findings to other populations must be done with caution; over $40 \%$ of 264 this population of LCV cases were geldings and the median duration of colic signs prior to 265 admission was 10 hours. The study only included two horses that underwent a large colon 266 resection and anastomosis (LCRA); in our hospital population LCRA has previously been associated with poor survival in horses with large intestinal disease [3]. However, Ellis et al. 
268 [9] demonstrated favourable survival in horses with strangulating LCV that underwent

269 LCRA, in a population comprising $88 \%$ mares with a mean duration of colic signs prior to

270 admission of 4 hours. Whether LCRA improves the prognosis of horses with strangulating

271 LCV requires further investigation $[9,11]$.

272

273 In conclusion LCV is associated with considerable post-operative mortality. Median

274 survival time in horses with a strangulating LCV that survived anaesthesia was 365 days.

275 Increased pre-operative PCV was significantly associated with increased post-operative

276 mortality, illustrating importance of early referral, prior to alteration in PCV. Alteration in

277 serosal colour intra-operatively, increased heart rate at 48 hours post-operatively, and colic

278 during post-operative hospitalisation were also significantly associated with increased post-

279 operative mortality. The study provides evidence based information on the survival of horses

280 with LCV and identifies parameters that may assist the clinician when determining the 281 prognosis for survival in individual horses.

282 


\begin{tabular}{|c|c|c|c|}
\hline Time post-operatively & Reason for death or euthanasia & $\begin{array}{l}\text { Number } \\
\text { of horses }\end{array}$ & $\begin{array}{l}\% \text { of } \\
\text { deaths }\end{array}$ \\
\hline \multirow[t]{4}{*}{ During surgery $(n=23)$} & Euthanased: non viable colon ${ }^{1}$ & 18 & 24 \\
\hline & Euthanased: unable to correct torsion & 3 & 4 \\
\hline & Euthanased: second LCV & 1 & 1.3 \\
\hline & Died under anaesthesia & 1 & 1.3 \\
\hline \multirow{2}{*}{$\begin{array}{l}\text { During recovery from } \\
\text { anaesthesia }(n=4)\end{array}$} & Euthanased: Unable to stand & 2 & 2.7 \\
\hline & Died: Unable to stand & 2 & 2.7 \\
\hline \multirow[t]{3}{*}{$\begin{array}{l}\text { During hospitalisation } \\
(n=26)\end{array}$} & $\begin{array}{l}\text { Euthanased: Systemic inflammatory response } \\
\text { syndrome (SIRS) / pain / colon necrosis }\end{array}$ & 23 & 30.7 \\
\hline & Euthanased: gastric rupture & 2 & 2.7 \\
\hline & Euthanased: concurrent synovial sepsis & 1 & 1.3 \\
\hline \multirow{3}{*}{$\begin{array}{l}\text { Following hospital } \\
\text { discharge }(n=22)\end{array}$} & Colic & 17 & 22.7 \\
\hline & Non-colic related & 3 & 4 \\
\hline & Unknown & 2 & 2.7 \\
\hline
\end{tabular}

285 Table 1. Reasons for death or euthanasia during intra and post-operative period. ${ }^{1}$ Non-viable

286 large colon as defined by surgeon at the time of exploratory laparotomy and recorded in the horse's surgical report.

\begin{tabular}{llllll}
\hline Variable & Coefficient & $\begin{array}{l}\text { Standard } \\
\text { error }\end{array}$ & $\begin{array}{l}\text { Hazard } \\
\text { ratio }\end{array}$ & $\begin{array}{l}\text { 95\% } \\
\text { Confidence } \\
\text { Intervals }\end{array}$ & p-value \\
\hline $\begin{array}{l}\text { PCV on admission } \\
(\%)\end{array}$ & 0.08 & 0.02 & 1.08 & $1.05-1.11$ & $<0.001$ \\
$\begin{array}{l}\text { PCV } * \ln (\text { time }) \\
\text { interaction effect }\end{array}$ & -0.04 & 0.01 & 0.96 & $0.95-0.97$ & $<0.001$ \\
\hline
\end{tabular}

289 Table 2. Final, multivariable Cox proportional hazards model (Model one) for post-operative 290 death in 116 horses undergoing anaesthesia and exploratory laparotomy for LCV, including a

$291 \quad \mathrm{PCV}^{*} \ln ($ time$)$ interaction term. 


\begin{tabular}{lll}
\hline Time (days) & Ln(t) & $\begin{array}{l}\text { Hazard } \\
\text { Ratio }\end{array}$ \\
\hline 1 & 0 & 1.08 \\
3 & 1.10 & 1.03 \\
5 & 1.61 & 1.01 \\
7 & 1.95 & 0.99 \\
\hline
\end{tabular}

294

295 Table 3. Calculation of the hazard ratio in the presence of a PCV*time interaction term at 296 different time points, with hazard ratio at time $t$ equal to $1.08 * 0.96^{\ln (t)}$.

\begin{tabular}{llllll}
\hline Variable & Coefficient & $\begin{array}{l}\text { Standard } \\
\text { error }\end{array}$ & $\begin{array}{l}\text { Hazard } \\
\text { ratio }\end{array}$ & $\begin{array}{l}\text { 95\% } \\
\text { Confidence } \\
\text { Intervals }\end{array}$ & p-value \\
\hline $\begin{array}{l}\text { Heart rate at 48 hours } \\
\text { post-operatively } \\
\text { (beats/minute) }\end{array}$ & 0.04 & 0.01 & 1.04 & $1.02-1.06$ & 0.0005 \\
$\begin{array}{l}\text { Serosal colour } \\
\text { (normal/abnormal) }\end{array}$ & 1.28 & 0.43 & 3.61 & $1.55-8.44$ & 0.003 \\
$\begin{array}{l}\text { Colic during post- } \\
\text { operative } \\
\text { hospitalisation }\end{array}$ & 0.97 & 0.50 & 2.63 & $1.00-6.95$ & 0.05 \\
\hline
\end{tabular}

300 Table 4. Final, multivariable Cox proportional hazards model (Model two) for post-

301 operative death in 89 horses with LCV recovering from general anaesthesia. 
303 Figure 1a and Figure 1b: Kaplan-Meier survival curves for a) 116 horses undergoing

304 general anaesthesia and exploratory laparotomy for LCV and b) 89 horses recovering from anaesthesia following exploratory laparotomy for LCV.

Figure 2: This graph illustrates the shape of the relationship between PCV on admission and the risk of mortality in 116 horses undergoing surgery for LCV. Spline fit (solid line), with 95\% confidence intervals (dotted lines) from univariable penalised Cox proportional hazards

310 regression model are illustrated.

312 Figure 3. Schoenfield residual plots for Model one: PCV. This graph reveals a lack of 313 proportionality over survival time, demonstrating that the effect of pre-operative PCV alters 314 with time $(\mathrm{p}<0.007)$.

316 Figure 4: This graph illustrates the shape of the relationship between heart rate at 48 hours

317 post-operatively and the risk of mortality in 89 horses with LCV that survived anaesthesia. 318 Spline fit (solid line), with 95\% confidence intervals (dotted lines) from univariable penalised 319 Cox proportional hazards regression model are illustrated.

\section{$321 \quad$ References}

322 1. Archer, D.C., Pinchbeck, G.L. and Proudman, C.J. (2011) Factors associated with survival of epiploic foramen entrapment colic: A multicentre, international study. Equine Veterinary Journal 43, 56-62. 
326 2. Proudman, C.J., Edwards, G.B., Barnes, J. and French, N.P. (2005) Factors affecting long-term survival of horses recovering from surgery of the small intestine. Equine Veterinary Journal 37, 360-365.

329

330 3. Proudman, C.J., Edwards, G.B., Barnes, J. and French, N.P. (2005) Modelling longterm survival of horses following surgery for large intestinal disease. Equine Veterinary Journal 37, 366-370.

333

4. Hardy, J. (2008) Specific diseases of the large colon. In: The Equine Acute Abdomen, Ed: N.A. White, Moore, J.N. and Mair, T.S., Teton NewMedia, Jackson. pp 639-642.

5. Proudman, C.J., Smith, J.E., Edwards, G.B. and French, N.P. (2002) Long-term survival of equine surgical colic cases. Part 1: Patterns of mortality and morbidity. Equine Veterinary Journal 34, 432-437.

6. Moore, R.M. (1996) Equine Large Colon Volvulus. In: Proceedings of American College of Veterinary Surgeons Annual Symposium. pp 236-237.

344 7. Mair, T.S. and Smith, L.J. (2005) Survival and complication rates in 300 horses undergoing surgical treatment of colic. Part 1: Short-term survival following a single laparotomy. Equine Veterinary Journal 37, 296-302. 
348 8. Snyder, J.R., Pascoe, J.R., Olander, H.J., Spier, S.J., Meagher, D.M. and Bleifer, D.R. (1989) Strangulating volvulus of the ascending colon in horses. Journal of the American Veterinary Medical Association 195, 757-764.

9. $\quad$ Ellis, C.M., Lynch, T.M., Slone, D.E., Hughes, F.E. and Clark, C.K. (2008) Survival and complications after large colon resection and end-to-end anastomosis for strangulating large colon volvulus in seventy-three horses. Veterinary Surgery 37, 786-790.

356

10. Southwood, L.L. (2004) Postoperative management of the large colon volvulus patient. Veterinary Clinics of North America - Equine Practice 20, 167-197.

11. Driscoll, N., Baia, P., Fischer Jr, A.T., Brauer, T. and Klohnen, A. (2008) Large colon

12. Dohoo, I., Martin, W. and Stryn, H. (2009) Modelling Survival Data. In: Veterinary Epidemiological Research, 2nd edn., Ed: I. Dohoo, Martin, W. and Stryn, H., AVC Inc., Charlotte Town, Prince Edward Island. pp 467-527.

13. Therneau, T.M., and Grambsch, P.M. (2000) Penalised models. In: Modelling Survival Data, Ed: T.M. Therneau, and Grambsch, P.M., Springer Verlag, New York. pp 120-126. 
372 14. Barton, M.H. and Collatos, C. (1999) Tumor necrosis factor and interleukin-6 activity and endotoxin concentration in peritoneal fluid and blood of horses with acute abdominal disease. Journal of veterinary internal medicine / American College of Veterinary Internal Medicine 13, 457-464.

15. Senior, J.M., Proudman, C.J., Leuwer, M. and Carter, S.D. (2011) Plasma endotoxin in horses presented to an equine referral hospital: Correlation to selected clinical parameters and outcomes. Equine Veterinary Journal 43, 585-591.

16. MacDonald, P.H., Dinda, P.K., Beck, I.T. and Mercer, C.D. (1993) The use of oximetry in determining intestinal blood flow. Surgery Gynecology and Obstetrics 176, 451-458.

17. Brusie, R.W., Sullins, K.E., Silverman, D.G. and Rosenberger, J.L. (1989) Fluorometric evaluation of large and small intestinal ischaemia in the horse. Equine Veterinary Journal 21, 358-363.

18. Mathis, S.C., Slone, D.E., Lynch, T.M., Hughes, F.E. and Clark, C.K. (2006) Use of colonic luminal pressure to predict outcome after surgical treatment of strangulating large colon volvulus in horses. Veterinary Surgery 35, 356-360.

19. Van Hoogmoed, L., Snyder, J.R., Pascoe, J.R. and Olander, H. (2000) Use of pelvic flexure biopsies to predict survival after large colon torsion in horses. Veterinary Surgery 29, 572-577. 
397 20. Van Hoogmoed, L., Snyder, J.R., Pascoe, J.R. and Olander, H.J. (2000) Evaluation of uniformity of morphological injury of the large colon following severe colonic torsion. Equine veterinary journal. Supplement, 98-100.

400

401

402

403

404

21. Levi, O., Affolter, V.K., Kass, P.H. and Le Jeunne, S.S. (2011) Retrospective evaluation of the use of pelvic flexure biopsy scores to predict short-term survival after large colon volvulus in 28 horses. In: 10th Equine Colic Research Symposium, Indianapolis, Indiana. pp 130-131.

405

22. Sykes, B.W. and Furr, M.O. (2005) Equine endotoxaemia - A state-of-the-art review of therapy. Australian Veterinary Journal 83, 45-50.

408

409

410

411

23. French, N.P., Smith, J., Edwards, G.B. and Proudman, C.J. (2002) Equine surgical colic: Risk factors for postoperative complications. Equine Veterinary Journal 34,

24. Fintl, C., Hudson, N.P.H., Mayhew, I.G., Edwards, G.B., Proudman, C.J. and Pearson, G.T. (2004) Interstitial cells of Cajal (ICC) in equine colic: An immunohistochemical study of horses with obstructive disorders of the small and large intestines. Equine Veterinary Journal 36, 474-479.

417

418 25. Swaminathan, R. and Brenner, H. (2011) Stastistical methods for cancer survival analysis. IARC scientific publications, 7-13. 
421 26. Hosgood, G. and Scholl, D.T. (2001) The effects of different methods of accounting for observations from euthanized animals in survival analysis. Preventive Veterinary Medicine 48, 143-154.

27. Stafford Johnson, M., Martin, M., Binns, S. and Day, M.J. (2004) A retrospective study of clinical findings, treatment and outcome in 143 dogs with pericardial effusion. Journal of Small Animal Practice 45, 546-552.

\section{Supporting Items}

430

Supporting item 1. Univariable associations of pre-operative categorical variables (Cox proportional hazards model) with post-operative death in 116 horses with a large colon volvulus $>360$ degrees undergoing exploratory laparotomy.

434

435 Supporting item 2. Univariable associations of pre-operative continuous variables (Cox 436 proportional hazards model) with post-operative death in 116 horses with a large colon 437 volvulus >360 degrees undergoing exploratory laparotomy.

438

439 Supporting item 3. Univariable associations of pre, intra and post-operative categorical 440 variables (Cox proportional hazards model) with post-operative death in 89 horses surviving 441 anaesthesia following surgery for large colon volvulus $>360$ degrees. 
443 Supporting item 4. Univariable associations of pre, intra and post-operative continuousl 444 variables (Cox proportional hazards model) with post-operative death in 89 horses surviving 445 anaesthesia following surgery for large colon volvulus $>360$ degrees.

446

447 Supporting item 5. Schoenfield residual plots for Model two: a) Heart rate at 48hours post448 operatively, b) Serosal colour and c) Colic during post-operative hospitalisation. These 449 graphs demonstrate proportionality throughout survival time, with all plots having no 450 significant pattern over time.

451

452 Supporting item 6a and Supporting item 6b. Log minus log cumulative hazard plots for 453 categorical variables in the final model Cox proportional hazards regression model (Model 454 two) for survival in 89 horses surviving anaesthesia following surgery with LCV. These 455 graphs demonstrate proportionality throughout survival time.

456

457

458

459

460

461 